\title{
ON SOME SOLUTIONS OF SECOND ORDER HYPERBOLIC DIFFERENTIAL EQUATIONS WITH CONSTANT COEFFICIENTS
}

\author{
by J. S. LOWNDES
}

(Received 22 January, 1986)

1. If we seek solutions of the hyperbolic differential equation

$$
\sum_{i=1}^{n} \frac{\partial^{2} u}{\partial x_{i}^{2}}+k^{2} u-\frac{\partial^{2} u}{\partial t^{2}}=0 \quad(k \geqslant 0)
$$

which depend only on the variables $t$ and $r=\left[\sum_{i=1}^{n} x_{i}^{2}\right]^{1 / 2}$, we see that these solutions must be even in $r$ and satisfy the differential equation

$$
T_{n}[u(r, t)]=\frac{\partial^{2} u}{\partial r^{2}}+\frac{(n-1)}{r} \frac{\partial u}{\partial r}+k^{2} u-\frac{\partial^{2} u}{\partial t^{2}}=0 .
$$

The object of this paper is to show that some recent results in the fractional calculus can be used to prove the following theorem.

THEOREM. For odd values of $n \geqslant 3$ and arbitrary functions $\phi$ with continuous derivatives up to the order $n-1$, the functions

$$
u(r, t)=T_{n}^{(n-3) / 2}\left[\int_{-1}^{1} J_{0}\left\{k r \sqrt{ }\left(1-\xi^{2}\right)\right\} \phi(t+\xi r) d \xi\right]
$$

are solutions of the differential equation

$$
T_{n}[u(r, t)]=0 .
$$

A corresponding result for the $n$-dimensional wave equation with rotational symmetry (i.e. equation (2) with $k=0$ ) is given in [1].

2. In what follows we shall make use of the generalized Erdélyi-Kober operator of fractional integration $\Im_{k}(\eta, \alpha)$ which is defined in [2] by

$$
\Im_{k}(\eta, \alpha) f(r)=2^{\alpha} k^{1-\alpha} r^{-2(\alpha+\eta)} \int_{0}^{r} x^{2 \eta+1}\left(r^{2}-x^{2}\right)^{(\alpha-1) / 2} J_{\alpha-1}\left\{k \sqrt{ }\left(r^{2}-x^{2}\right)\right\} f(x) d x,
$$

where $r>0, \alpha>0, k \geqslant 0$ and $J_{\alpha-1}$ is the Bessel function of the first kind.

A useful result connecting the above operator with the singular differential operator

$$
L_{\eta}=\frac{\partial^{2}}{\partial r^{2}}+\frac{(2 \eta+1)}{r} \frac{\partial}{\partial r}
$$

is contained in the following lemma [2].

Glasgow Math. J. 29 (1987) 69-72. 
Lemma. If $\alpha>0, f(r) \in C^{2}(0, b)$ for some $b>0, r^{2 \eta+1} f(r)$ is integrable at the origin and $r^{2 \eta+1} f^{\prime}(r) \rightarrow 0$ as $r \rightarrow 0+$, then

$$
\Im_{k}(\eta, \alpha) L_{\eta} f(r)=\left(L_{\eta+\alpha}+k^{2}\right) \Im_{k}(\eta, \alpha) f(r) .
$$

3. Adopting the notation of (6) we see that the one-dimensional wave equation

$$
L_{-1 / 2} w-\frac{\partial^{2} w}{\partial t^{2}}=0
$$

with the conditions

$$
w(0, t)=2 \phi(t), \quad \frac{\partial}{\partial r} w(0, t)=0
$$

has the solution

$$
w(r, t)=\phi(t+r)+\phi(t-r),
$$

for arbitrary differentiable functions $\phi$.

We now introduce the function

$$
w_{\alpha}(r, t)=\frac{\Gamma\left(\alpha+\frac{1}{2}\right)}{\Gamma\left(\frac{1}{2}\right)} \Im_{k}\left(-\frac{1}{2}, \alpha\right) w(r, t) \quad(\alpha>0)
$$

and apply the operator $\left[\Gamma\left(\frac{1}{2}\right)\right]^{-1} \Gamma\left(\alpha+\frac{1}{2}\right) \Im_{k}\left(-\frac{1}{2}, \alpha\right)$ to equations (8), (9) and (10). In this way, on using the result (7) of the lemma, we find that the solution of the differential equation

$$
T_{2 \alpha+1}\left[w_{\alpha}(r, t)\right]=0 \quad(\alpha>0)
$$

with the conditions

$$
w_{\alpha}(0, t)=2 \phi(t), \quad \frac{\partial}{\partial r} w_{\alpha}(0, t)=0
$$

is given by

$$
\begin{aligned}
w_{\alpha}(r, t) & =\frac{\Gamma\left(\alpha+\frac{1}{2}\right)}{\Gamma\left(\frac{1}{2}\right)} \Im_{k}\left(-\frac{1}{2}, \alpha\right)[\phi(t+r)+\phi(t-r)] \\
& =2^{\alpha} \frac{\Gamma\left(\alpha+\frac{1}{2}\right)}{\Gamma\left(\frac{1}{2}\right)}(k r)^{1-\alpha} \int_{-1}^{1}\left(1-\xi^{2}\right)^{(\alpha-1) / 2} J_{\alpha-1}(\rho) \phi(t+\xi r) d \xi,
\end{aligned}
$$

where $\rho=k r V\left(1-\xi^{2}\right)$.

With the above results we can write

$$
\begin{aligned}
T_{n}\left[w_{\alpha}(r, t)\right] & =T_{2 \alpha+1}\left[w_{\alpha}(r, t)\right]+\frac{(n-2 \alpha-1)}{r} \frac{\partial}{\partial r} w_{\alpha} \\
& =\frac{(n-2 \alpha-1)}{r} \frac{\partial}{\partial r} w_{\alpha}
\end{aligned}
$$


and from equations (14) and (15) we find that

$$
\begin{aligned}
T_{n}\left[w_{\alpha}(r, t)\right]= & 2^{\alpha} \frac{\Gamma\left(\alpha+\frac{1}{2}\right)}{\Gamma\left(\frac{1}{2}\right)} \frac{(n-2 \alpha-1)}{r}\left\{-k(k r)^{1-\alpha} \int_{-1}^{1}\left(1-\xi^{2}\right)^{\alpha / 2} J_{\alpha}(\rho) \phi(t+\xi r) d \xi\right. \\
& \left.+(k r)^{1-\alpha} \int_{-1}^{1} \xi\left(1-\xi^{2}\right)^{(\alpha-1) / 2} J_{\alpha-1}(\rho) \phi^{\prime}(t+\xi r) d \xi\right\}
\end{aligned}
$$

On performing an integration by parts on the last integral in the above equation we get

$$
T_{n}\left[w_{\alpha}(r, t)\right]=2^{\alpha} \frac{\Gamma\left(\alpha+\frac{1}{2}\right)}{\Gamma\left(\frac{1}{2}\right)} \frac{(n-2 \alpha-1)}{(k r)^{\alpha}} \int_{-1}^{1}\left(1-\xi^{2}\right)^{\alpha / 2} J_{\alpha}(\rho)\left[\phi^{\prime \prime}(t+\xi r)-k^{2} \phi(t+\xi r)\right] d \xi
$$

and with the aid of this result we can now prove the theorem.

4. Proof of the theorem. When $\alpha=1$ the solution of equations (12) and (13) is given by

$$
w_{1}(r, t)=\int_{-1}^{1} J_{0}(\rho) \phi(t+\xi r) d \xi
$$

where $\rho=k r \sqrt{ }\left(1-\xi^{2}\right)$.

Using the result (17) we have

$$
T_{n}\left[w_{1}(r, t)\right]=\frac{(n-3)}{k r} \int_{-1}^{1}\left(1-\xi^{2}\right)^{1 / 2} J_{1}(\rho)\left[\phi^{\prime \prime}(t+\xi r)-k^{2} \phi(t+\xi r)\right] d \xi
$$

and repeated applications of the formula (17) yield the expression

$$
T_{n}^{m}\left[w_{1}(r, t)\right]=\frac{(n-3)(n-5) \ldots(n-2 m-1)}{(k r)^{m}} \int_{-1}^{1}\left(1-\xi^{2}\right)^{m / 2} J_{m}(\rho) \Phi_{m}(t+\xi r) d \xi
$$

when $n \geqslant 2 m+1$,

$$
\Phi_{m}(t+\xi r)=\sum_{s=0}^{m}(-1)^{m-s}\left(\begin{array}{c}
m \\
s
\end{array}\right) k^{2(m-s)} \phi^{(2 s)}(t+\xi r)
$$

and $\phi$ is any function with continuous derivatives up to order $2 m$.

In this way we find that, for odd values of $n \geqslant 3$,

$$
T_{n}^{(n-1) / 2}\left[w_{1}(r, t)\right]=T_{n}\left\{T_{n}^{(n-3) / 2}\left[w_{1}(r, t)\right]\right\}=0
$$

and this proves the theorem. 
5. In order to construct a simple example we take $\phi(t)=e^{i \beta t}$ and in this case we see that equation (18) gives

$$
\begin{aligned}
w_{1}(r, t) & =\int_{-1}^{1} J_{0}\left\{k r \sqrt{ }\left(1-\xi^{2}\right)\right\} e^{i \beta(t+\xi r)} d \xi \\
& =2 e^{i \beta t} \int_{0}^{1} J_{0}\left\{k r \sqrt{ }\left(1-\xi^{2}\right)\right\} \cos (\xi \beta r) d \xi \\
& =2 e^{i \beta t} \frac{\sin (a r)}{a r}
\end{aligned}
$$

where $a=\sqrt{ }\left(\beta^{2}+k^{2}\right)$ and the integral has been evaluated by a result given in [3].

Using the theorem we have that, for odd values of $n \geqslant 3$, the functions

$$
v_{n}(r, t)=T_{n}^{(n-3) / 2}\left[2 e^{i \beta z} \frac{\sin (a r)}{a r}\right]
$$

satisfy the differential equation

$$
T_{n}\left[v_{n}(r, t)\right]=0 .
$$

As two special cases it can easily be shown that when $n=5$,

$$
v_{5}(r, t)=4 e^{i \beta t}\left[\frac{\cos (a r)}{r^{2}}-\frac{\sin (a r)}{a r^{3}}\right]
$$

and when $n=7$,

$$
v_{7}(r, t)=16 e^{i \beta t}\left[\frac{3 \sin (a r)}{a r^{5}}-\frac{3 \cos (a r)}{r^{4}}-\frac{a \sin (a r)}{r^{3}}\right],
$$

which are even functions of the variable $r$.

\section{REFERENCES}

1. R. Courant and D. Hilbert, Methods of mathematical physics, Vol. 2 (Interscience, 1962). $35-41$.

2. J. S. Lowndes, An application of some fractional integrals, Glasgow Math. J. 20 (1979),

3. W. Magnus, F. Oberhettinger and R. P. Soni, Formulas and theorems for the special functions of mathematical physics, 3rd. ed. (Springer-Verlag, 1966).

DepartMent of MATHEMATics

UNIVERSITY OF STRATHCLYDE

GLASGOW G1 1XH 